\title{
E-rara.ch: eine Schweizer Erfolgsgeschichte
}

\begin{abstract}
E-rara.ch ist eine mit nationaler Unterstützung aufgebaute Plattform für digitalisierte Dokumente aus der Zeit des 15. bis Anfang des 20. Jahrhunderts, die aus einer Reihe Schweizer Bibliotheken kommen. Ursprünglicher Ausgangspunkt war die Digitalisierung von Werken des 16. Jahrhunderts mit Schweizer Druckorten, doch hat sich das Spektrum mittlerweile erheblich ausgeweitet. Mit mehr als 57’000 online zugänglichen Titeln bietet die Plattform heute sowohl für wissenschaftlich interessierte Personen als auch für die breite Öffentlichkeit einen einfachen Zugang nicht nur zu digitalisierten alten Drucken, sondern auch zu sonstigen illustrierten Materialien sowie zu Karten und Musikdrucken.

Bereits nach kurzer Laufzeit im Anschluss an die offizielle Lancierung im März 2010 hat sich die Plattform e-rara.ch im In- und Ausland als Erfolgsgeschichte etabliert.
\end{abstract}

Somit ist nach nunmehr sieben Jahren Betrieb ein guter Zeitpunkt, eine Zwischenbilanz zu ziehen.

\section{Von der Idee zum Ziel: eine gemeinsame digitale Zukunft}

Seit Ende der 1990er Jahre haben sich die Schweizer Hochschulbibliotheken mit dem Thema elektronische Angebote als relevante Informationsquellen für Wissenschaft, Forschung und Lehre befasst. ${ }^{1}$ Dies war der erste Schritt zur Idee, das Projekt Elektronische Bibliothek der Schweiz ins Leben zu rufen. Da es für bibliotheksspezifische Förderprojekte keine geeigneten Instrumente gab, konnte mit e-lib.ch (2008-2011) ${ }^{2}$ eine grosse Lücke beim Ausbau der Informationslandschaft der Schweiz geschlossen werden. Im Rahmen der entsprechenden Diskussionen vor dem Jahr 2008 wurde darüber hinaus auch deutlich, dass in der Schweiz, im Gegensatz zur Situation in den Nachbarländern, bezüglich des Themas der retro-

1 Wolfram Neubauer: Schweizer Bibliotheken im Netz: Stand und Zukunft digitaler Angebote. In: Bibliothek Forschung und Praxis 36 (2012), S. 70-77.

2 Matthias Töwe: Elektronische Bibliothek Schweiz E-LIB.CH - Umfeld und Inhalte des landesweiten Projekts. In: Mitteilungen der VÖB 62/1 (2009), S. 51-64; Susanne Benitz, Nadja Böller: e-lib.ch: Elektronische Bibliothek Schweiz - Eine Erfolgsgeschichte? In: b.i.t.online 16/2 (2013), S. 97-102.

Ә Open Access. () Meda Diana Hotea, publiziert von De Gruyter. (c) BY-NC-ND Dieses Werk ist lizenziert unter der Creative Commons Attribution-NonCommercial-NoDerivatives 4.0 Lizenz. https://doi.org/10.1515/9783110553796-026 
spektiven Digitalisierung wertvoller Medienbestände erheblicher Nachholbedarf erkennbar war. Mit den durch das Projekt e-lib.ch bereitgestellten Fördermitteln erhielten die Wissenschaftlichen Bibliotheken des Landes die notwendigen Impulse, durch den Aufbau von Digitalisierungsplattformen und Fachportalen ${ }^{3}$ diese unerfreuliche Situation auszugleichen.

Unter dem Titel „www.e-rara.ch: die Online-Plattform für digitalisierte Rara aus Schweizer Bibliotheken“ wurde im August 2007 der Projektantrag für die Digitalisierung alter Drucke des 16. Jahrhunderts mit Druckorten in der Schweiz bei e-lib.ch eingereicht. Die wichtigen Projektziele waren einmal der Aufbau entsprechender Digitalisierungskompetenzen bei den Partnerbibliotheken, dann die eigentliche Digitalisierung von Inhalten und schliesslich der Aufbau einer nachhaltigen Online-Plattform für die Präsentation der digitalisierten Schweizer Drucke.

Die Federführung lag vom Start weg bei der ETH-Bibliothek: Hier wurde der Projektantrag konzipiert und ausgearbeitet und darüber hinaus erfolgen die Projektleitung, das Hosting und der Betrieb der neuen Plattform bis heute an der ETHBibliothek. Allerdings wirkten eine Reihe weiterer Bibliotheken von Beginn an am Projekt mit. So haben sich die Zentralbibliothek Zürich (aus dem NEBIS-Verbund), die Universitätsbibliotheken Basel und Bern (aus dem IDS-Verbund Basel/Bern) sowie die Bibliothèque de Genève (aus dem RERO-Verbund) bereit erklärt, sich aktiv für den Aufbau der neuen Plattform einzusetzen. Damit konnten bereits bei diesem Projekt die Sprach- und Verbundgrenzen (anfangs zwischen dem deutschen und französischen Teil der Schweiz) überwunden werden und die Voraussetzungen für ein landesweites Kooperationsprojekt sowie für eine nationale Plattform der digitalisierten alten Drucke waren erfüllt. ${ }^{4}$ Eingereicht wurde der Projektantrag im Rahmen von e-lib.ch bei der damaligen CRUS ${ }^{5}$ im Jahr 2007, wobei die geplante Projektlaufzeit von 2008 bis zum Jahr 2012 reichen sollte. Die ersten beiden Jahre waren geprägt vom Aufbau der administrativen Organisation sowie vom technischen Aufbau der Plattform. Da e-lib.ch als Kooperationsprojekt

3 Erwähnt werden sollen an dieser Stelle das digitale Schweizer Zeitschriftenarchiv; damals als retro.seals.ch bekannt, heute in e-periodica.ch (http://www.e-periodica.ch/) umbenannt oder Kartenportal.CH, das Fachportal für Karten und Geodaten (http://www.kartenportal.ch/). Alle Links in diesem Beitrag wurden am 26.03.2018 überprüft.

4 Franziska Geisser: e-rara.ch: ein Schweizer Digitalisierungsprojekt mit internationaler Ausstrahlung. In: arbido 3/2011, S. 23-26; Alexis Rivier: e-rara.ch, une bibliothèque numérique pour les livres anciens. In: RESSI 15/2014, http://www.ressi.ch/num15/article_102.

5 Die CRUS, die Conférence des Recteurs des Universités Suisses (Rektorenkonferenz der Schweizer Universitäten), existierte von 2001 bis 2014 und ist in der neuen Organisation swissuniversities aufgegangen. 
angelegt war, spiegelte sich diese Situation auch in der Projektorganisation wieder. Die Projektleitung arbeitete eng mit den lokalen Koordinatorinnen und Koordinatoren an den einzelnen Partnerinstitutionen zusammen, wobei sich aus den vier geplanten Teilprojekten Inhalte, Digitalisierung, Metadaten und Online-Plattform jeweils entsprechende Arbeitsgruppen entwickelt haben.

Die Beantwortung der Frage nach einer geeigneten Präsentationsplattform war bereits im Projektantrag ein wichtiges Element, so dass bereits dort wesentliche Anforderungskriterien formuliert wurden. So sollte das Softwareprodukt flexibel und ausbaubar sein, einfachen Zugriff auf verschiedene Bibliothekskataloge bieten, eine sinnvolle Steuerung des Digitalisierungsworkflows ermöglichen und nicht zuletzt ein harmonisches Arbeitsumfeld mit einem zentralen Betrieb und einer dezentralen Bearbeitung der Inhalte sichern. Letztendlich fiel die Entscheidung dann auf das Produkt Visual Library, das die formulierten Anforderungen weitgehend erfüllte.

Mit einem ersten Set von etwa 500 digitalisierten Titeln wurde die Plattform e-rara.ch am 15. März 2010 online aufgeschaltet.

\section{E-rara.ch als bibliothekarische Herausforderung}

Unter dem Druck und Anspruch der technischen Entwicklungen der letzten 10 bis 15 Jahre haben sich die Wissenschaftlichen Bibliotheken wesentlich modernisiert und sich mit den veränderten Anforderungen zurechtgefunden. Somit hat für die Wissenschaftlichen Bibliotheken eine neue Ära begonnen, die einmal bestimmt wird durch die Entwicklungen aus der Informations- und Kommunikationswelt und zum zweiten durch die sich daraus ergebenden neuen Anforderungen aus Wissenschaft, Forschung und Lehre. Diese Situation gilt selbstverständlich auch für die klassischen Sammlungen von Handschriften, Alten Drucken, Bildmaterialien, Karten und Objekten, die sich alle als wesentliche Bestände in Wissenschaftlichen Bibliotheken befinden. Somit ist es nicht überraschend, dass auch über die zukünftige Rolle der Sammlungen in den Bibliotheken ein intensiver und kritischer Meinungsaustausch begonnen hat. ${ }^{6}$ In dieser zukunftsorientierten Diskussion spielt natürlich der Aspekt der Digitalisierung von Altbeständen und deren Präsentation über entsprechende technische Plattformen eine wesentliche Rolle. Von dieser Diskussion sind selbstverständlich auch die Schweizer Bibliotheken nicht unberührt geblieben.

6 Daniel Traister: Is there a future for special collections? And should there be? In: RBM: A Journal of Rare Books, Manuscripts, and Cultural Heritage 1/1 (2000), S. 54-76. 
Der Aufbau der neuen Plattform hat des Weiteren erhebliche Auswirkungen auf die teilnehmenden Bibliotheken gehabt. Dies betrifft sowohl die technischen Infrastrukturen wie auch die Betriebsorganisation. Klassische bibliothekarische Arbeitsplätze mit dem vertrauten Mobiliar und Bücherwagen wurden ergänzt durch das Digitalisierungszentrum mit komplizierten manuellen Scannern, mit Scanrobotern und hochkomplexen Kameras. Dazu kommen neue, bis dahin undenkbare Arbeitsinhalte und neue Workflows. Alle fünf ,Gründungspartner haben sich (neben ihren klassischen Aufgaben) in Digitalisierungsstandorte für das Projekt e-rara.ch umgewandelt.

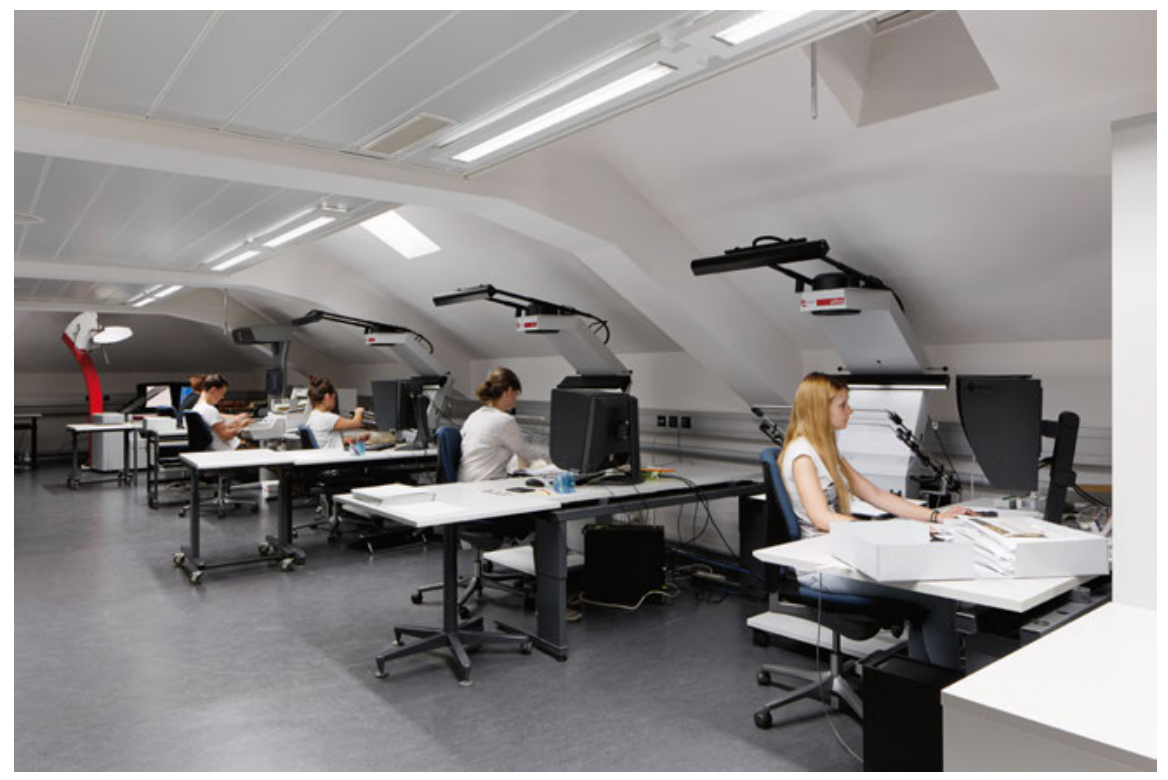

Abb. 1: das neue Digitalisierungszentrum der ETH-Bibliothek, 13.07.2015 ${ }^{7}$

In diesen fünf Digitalisierungszentren werden heute nicht nur die eigenen Altbestände digitalisiert, sondern auch die anderer Schweizer Bibliotheken, die sich an das Projekt e-rara.ch angeschlossen haben und die notwendige technische Infrastruktur nicht vorhalten können. Hieraus wiederum ist für die involvierten Institutionen eine komplexe logistische Herausforderung entstanden. Die Organisation des Transportes der wertvollen Bestände, deren sichere Aufbewahrung und nicht zuletzt die konservatorischen Hürden während des Digitalisierungs-

7 ETH-Bibliothek Zürich, Bildarchiv / Photograph: Blaser, Frank / KOM_000273 / CC BY-SA 4.0. 
prozesses haben diese Medien wieder mehr in das Blickfeld der Bibliotheksarbeit gerückt. Die teilnehmenden Projektpartner waren nicht nur mit der Auswahl der technischen Infrastruktur und mit den logistischen Problemen konfrontiert, sondern mussten sich beispielsweise auch mit Fragen der Scanqualität befassen, die die international bestehenden Qualitätsstandards einhalten, wenn nicht übertreffen sollten. Da im benachbarten Deutschland Regeln ${ }^{8}$ für die Mediendigitalisierung bereits durch die entsprechende Fördereinrichtung (DFG) durchgesetzt worden waren, wurden diese in die hiesigen Workflows als Orientierungshilfe übernommen. Auf Basis der nunmehr langjährigen Erfahrungen beim Scannen von Bibliotheksmaterialien hat jedoch beispielsweise auch die ETH-Bibliothek Best Practice-Regeln entwickelt, die für andere Bibliotheken vorbildlich sein können. ${ }^{9}$

Die doppelte Vorhaltung von analogen und digitalisierten (virtuellen) Beständen und hier insbesondere die Titelauswahl für die Digitalisierung hat sich im Projektverlauf als eine erhebliche Herausforderung herausgestellt. Wie bereits eingangs erwähnt, war es zu Beginn des Projektes das erklärte Ziel, Schweizer Drucke des 16. Jahrhunderts zu digitalisieren und auf einer geeigneten Präsentationsplattform aufzuschalten. An dieser Stelle ist die Arbeit der Arbeitsgruppe „Inhalte“ unter der Federführung der Zentralbibliothek Zürich besonders zu erwähnen. So wurde eine Bestandsaufnahme der Schweizer Druckorte durchgeführt und die vorhandenen Titel des 16. Jahrhunderts wurden ermittelt. Diese Titel wurden dann von den Partnerbibliotheken prioritär digitalisiert. Diesem ursprünglichen Ziel folgt e-rara.ch im Prinzip auch noch heute, wofür die Digitalisierung der unikalen Bestände der Zentralbibliothek Solothurn ${ }^{10}$ ein gutes Beispiel ist. Hierzu parallel haben sich allerdings auch die Interessen der Gründungsinstitutionen ausgeweitet und die ursprüngliche Beschränkung auf das 16. Jahrhundert wurde aufgegeben, so dass heute der Digitalisierungszeitraum bis zum Beginn des 20. Jahrhunderts reicht. Somit hat sich e-rara.ch im Laufe der Jahre zu einer allgemeinen Digitalisierungsplattform für Medienbestände aus Schweizer Bibliotheken entwickelt und von der ursprünglichen Beschränkung auf Alte Drucke verabschiedet.

Dies lässt sich gut dokumentieren durch die erstmalige Aufschaltung von Karten im Jahre 2012 und mit den Entwicklungen, die sich aus dem Projekt

8 Die genannten Praxisregeln für die Digitalisierung sind auf der Webseite der Deutschen Forschungsgemeinschaft (DFG) veröffentlicht: http://www.dfg.de/formulare/12_151/12_151_de.pdf.

9 In diesem Zusammenhang siehe das Dokument Best Practices Digitalisierung der ETH-Bibliothek: http://www.library.ethz.ch/de/ms/DigiCenter.

10 Diese Bestände sind seit Anfang des Jahres 2017 recherchierbar unter http://www.e-rara.ch/ solothurn/nav/classification/14984518. 
DigiTUR der Zentralbibliothek Zürich ergeben haben. Mit Unterstützung des Lotteriefonds des Kantons Zürich hat die Zentralbibliothek ein grosses Digitalisierungsunternehmen initiiert, mit dem seit dem Jahr 2013 wichtige Teile des kulturellen Erbes von Stadt und Kanton Zürich digitalisiert werden sollten. Der voraussichtliche Abschluss des Projektes mit der Publikation der digitalisierten Dokumente auf den Plattformen e-rara.ch und e-manuscripta.ch ${ }^{11}$ ist für das Frühjahr 2018 vorgesehen. Auf e-rara.ch wurden im Kontext von DigiTUR mittlerweile 13'000 alte Drucke, mehr als 12'000 Titel illustriertes Material, mehr als 2'500 Karten und etwa 600 Musikdrucke aufgeschaltet und somit online zugänglich gemacht. Hiermit hat e-rara.ch zum Ende des Jahres 2016 die Grenze von 50 '000 teilweise im Volltext recherchierbaren Dokumenten überschritten und einen weiteren Meilenstein erreicht.

\section{Die Plattform e-rara.ch im Jahr 2017}

Seit der erstmaligen Aufschaltung von e-rara.ch hat sich die Projektleitung intensiv darum bemüht, kontinuierlich neue Partner zu gewinnen und dies mit Erfolg: Die Zahl der beteiligten Einrichtungen hat sich mittlerweile auf 13 erhöht. Waren zu Beginn des Projektes mehr oder weniger nur grosse wissenschaftliche (Universitäts-)Bibliotheken vertreten, hat sich das Spektrum mittlerweile ausgeweitet. Gleichzeitig sind nunmehr durch die Einbindung der Biblioteca Salita dei Frati und der Biblioteca SUPSI DFA aus dem Tessin alle Landesteile vertreten, so dass die erwünschte nationale Ausstrahlung erreicht werden konnte. Andererseits gibt es jedoch noch eine Reihe weiterer potenzieller Partnereinrichtungen in der Schweiz, die bisher noch nicht dabei sein können. Hier sind besonders Stadt- und Kantonsbibliotheken zu erwähnen, die nicht selten über qualitativ und quantitativ bemerkenswerte Altbestände verfügen, deren Einbindung in e-rara.ch äusserst wünschenswert wäre. Hier bleibt also für die nächsten Jahre noch einiges zu tun.

11 Bei e-manuscripta.ch handelt es sich um eine Plattform für digitalisierte handschriftliche Quellen aus Schweizer Bibliotheken und Archiven. Die Applikation ist mit e-rara.ch nah verwandt und läuft ebenfalls unter dem Softwaretool Visual Library. Siehe dazu den Beitrag von Alexa Renggli in diesem Band. 


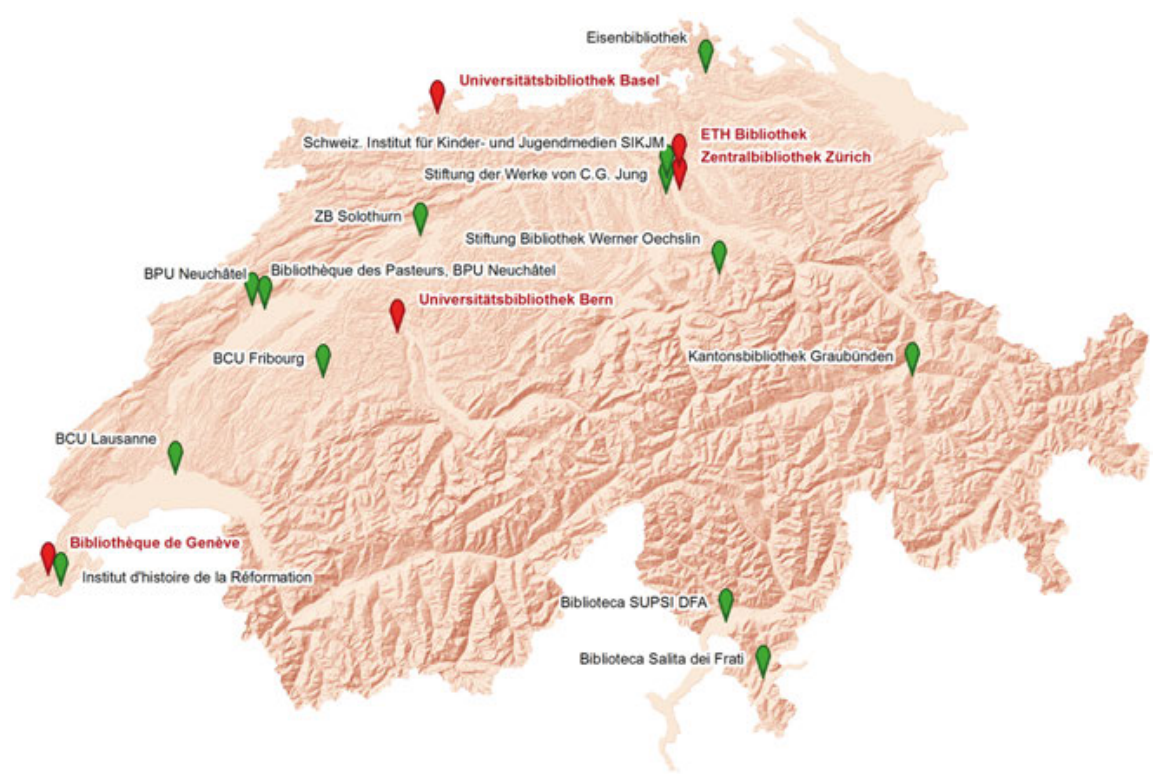

Abb. 2: e-rara.ch auf nationaler Ebene

Die Plattform e-rara.ch hat sich mittlerweile nicht nur landesweit weiterentwickelt, sondern auch die entsprechenden Nutzungszahlen sind exponentiell angestiegen. Wurden im Jahr 2010 etwa 208'000 Visits verzeichnet, sind die Zahlen bis Ende 2016 auf 1'175'000 angewachsen.

$\mathrm{Zu}$ diesem grossen Erfolg haben allerdings nicht nur die hochwertige Scanqualität und die Vielfalt der angebotenen Inhalte beigetragen, sondern auch die kontinuierlichen Bemühungen, die Plattform noch attraktiver und nutzerfreundlicher zu machen.

Im Jahr 2015 fiel die Entscheidung, über Open Data einen offenen Zugang zu allen Dokumenten der Plattform e-rara.ch zu unterstützen. Hierfür wurden die Nutzungsbedingungen angepasst und die Digitalisate mit einer Public DomainLizenz versehen oder unter Creative Commons BY-SA 4.0 lizenziert. Diese Dokumente können somit für wissenschaftliche, private, nicht-kommerzielle und kommerzielle Zwecke frei verwendet werden.

Der Gedanke, den Zugriff auf die Plattform kontinuierlich zu verbessern, brachte es mit sich, auch über die Möglichkeit einer Texterkennung nachzudenken. Bis zum heutigen Tage stellt die Diversität der verwendeten Schriften in den alten Drucken bei deren Rezeption ein nicht zu unterschätzendes Problem dar, so dass es nur naheliegend war, sich auch mit diesem Thema zu befassen. Um diese Situation zu verbessern, wurde im Jahre 2015 beim Förderprogramm SUK 
P-2 ${ }^{12}$ ein Antrag lanciert, mit dem die Möglichkeit und Sinnhaftigkeit der Einführung einer Texterkennung für mit Antiquaschrift gedruckte Dokumente aus dem Zeitraum zwischen den Jahren 1830 bis zum Anfang des 20. Jahrhundert erarbeitet werden sollte. Das Projekt e-rara.ch: Volltext wurde Mitte 2016 gestartet und kann im Herbst dieses Jahres erfolgreich abgeschlossen werden. Federführend ist hier wiederum die ETH-Bibliothek unter intensiver Mitwirkung der Universitätsbibliotheken Basel und Bern, der Zentralbibliothek Zürich und der Bibliothèque de Genève. ${ }^{13}$ Auch bei diesem Projekt haben sich die Antragsteller an einem Projekt der Deutschen Forschungsgemeinschaft ${ }^{14}$ orientiert.

Blicken wir jetzt in die Zukunft, dann sind die Konturen der Weiterentwicklung von e-rara.ch bereits am Horizont erkennbar. Neben der Aufgabe, kontinuierlich weitere Partner für die Plattform zu gewinnen, werden folgende Aspekte die Arbeit der nächsten Monate und Jahre bestimmen: die Prüfung der Möglichkeit, für weitere Schrifttypen eine Volltexterkennung zu erarbeiten, und die Einführung neuer Technologien wie etwa IIIF (International Image Interoperability Framework). Zum zehnjährigen Jubiläum der Plattform im Frühjahr 2020 werden hoffentlich weitere nützliche Applikationen in e-rara.ch integriert sein.

Zusammenfassend lässt sich somit festhalten, dass e-rara.ch sowohl für Wissenschaft, Forschung und Lehre, aber auch für die breite Öffentlichkeit eine sinnvolle und gleichzeitig erfolgreiche Applikation der Schweizer wissenschaftlichen Bibliotheken darstellt.

Tab. 1: e-rara.ch im chronologischen Überblick

\begin{tabular}{ll}
\hline August 2007 & Einreichung des Projektantrages bei e-lib.ch \\
\hline November 2007 & Genehmigung des Projektes durch die CRUS \\
\hline 15. März 2010 & Online-Aufschaltung erster Dokumente auf der Plattform \\
\hline 2012-2013 & Verlängerung des Finanzierungszeitraums für das Projekt \\
\hline Januar 2013 & Relaunch der Webseite, der Navigation und der Recherche der Plattform \\
\hline Ende 2013 & Abschluss des Projektes e-rara.ch \\
\hline Januar 2014 & $\begin{array}{l}\text { kooperativer Betrieb der Plattform durch die Gründungsinstitutionen, } \\
\text { Verabschiedung eines Business- und Kostenmodels }\end{array}$ \\
\hline
\end{tabular}

12 Beim hier relevanten Förderprogramm handelt es sich um das Programm „Wissenschaftliche Information: Zugang, Verarbeitung und Speicherung“(https://www.swissuniversities.ch/de/).

$13 \mathrm{http} / /$ www.library.ethz.ch/de/Ueber-uns/Projekte/e-rara.ch-Volltext.

14 http://ocr-d.de/. 
Einreichung eines weiteren Projektantrages beim Förderprogramm SUK P-2 (e-rara.ch: Volltext)

\section{Ende 2016} Anpassung der Nutzungsbedingungen: e-rara.ch öffnet sich für Open Data

Januar 2017 neue Organisationsstruktur für den Betrieb der Plattform; Auflösung der bis dahin geltenden Projektorganisation

September 2017 Abschluss des Projektes e-rara.ch: Volltext

2018-2019 weitere Entwicklungen der Plattform

15. März 2020 zehnjähriges Jubiläum 